\title{
Drug Delivery: Plant Lectins as Bioadhesive Drug Delivery Systems
}

\author{
Marija Gavrovic-Jankulovic, Radivoje Prodanovic
}

Department of Biochemistry, Faculty of Chemistry, University of Belgrade, Belgarde, Serbia.

E-mail: mgavrov@chem.bg.ac.rs

Received September $17^{\text {th }}, 2011$; revised October $9^{\text {th }}$, 2011; accepted November $11^{\text {th }}, 2011$.

\begin{abstract}
Selective targeting of drugs to the proposed site of action provides therapeutic advantages such as reduced toxicity and smaller dose levels. Despite a huge progress made in drug design and delivery systems, many challenges still have to be solved. Small therapeutic drugs always have the potential to pass into the kidneys and be excreted from the body. The use of macromolecular constructs (carriers) that allow longer circulation times, contribute to improved chemical functionality and more precise drug delivery is an attractive alternative option. Bioadhesive systems which will utilize intense contact to increase the drug concentration gradient could be an attractive approach. Because of their specific carbohydrate-binding, lectins can interact with glycoconjugates present on the epithelial cells that line all of the organs exposed to the external environment. The unique carbohydrate specificities of plant lectins can facilitate mucoadhesion and cytoadhesion of drugs. As immunostimulatory molecules with an adjuvant effect plant lectins can also be employed in vaccine development.
\end{abstract}

Keywords: Drug Delivery, Epithelium, Plant Lectin, Polymers, Vaccine

\section{Introduction}

The traditional routes of drug administration are nasal, oral, subcutaneous, intramuscular, intravenous, topical, ophthalmic and rectal. A wide variety of polymeric biomaterials are compounds of various formulations and devices which are routinely used for delivering drug to the body. However, these medication delivery systems may not always achieve optimal drug concentration at the appropriate site, nor do they necessarily minimize local or systemic toxicity [1]. Therefore, there has been enormous interest in developing controlled-release formulations and devices that can maintain a desired blood plasma level of the drug for longer periods of time without reaching a toxic level or dropping below the minimum effective level [2].

This paper will give an overview of the systems that have been used for drug delivery via mucosal surfaces and will showcase recent employment of plant-derived lectins in creation of drug delivery vehicles, but also their immunomodulatory potential.

\section{Mucoadhesive Polymer Drug Delivery Systems}

Mucoadhesive drug delivery systems are vehicles which utilize the property of bioadhesion of certain polymers that become adhesive on hydration [3] and can be used for targeting a drug to the particular region of the body for an extended period of time [4]. In the case of a polymer attached to the mucin layer of a mucosal tissue the term "mucoadhesion" is employed [5].

The polymeric properties which are relevant for high retention via mucoadhesive interactions at particular targeted sites include hydrophobicity, negative charge potential and the presence of hydrogen bond forming groups [6]. In addition, the polymer should possess sufficient flexibility to penetrate the mucus network and be biocompatible, non-toxic and economically favorable [7]. According to Park and Robinson, [8] polymers commonly employed in the manufacturing of mucoadhesive drug delivery platforms that adhere to mucinepithelial surfaces are categorized as follows:

- Polymers that are bioadhesive due to their stickiness,

- Polymers that adhere through nonspecific, noncovalent, primarily electrostatic interactions,

- Polymers that bind to a specific receptor on the cell surface.

\subsection{First-Generation Mucoadhesive Polymers}

According to the net overall charge, mucoadhesive poly- 
mers have been divided into three categories: anionic polymers, cationic polymers, and non-ionic polymers, with the first two groups exhibiting the highest mucoadhesive strength [9]. Due to their high mucoadhesive potential and low toxicity anionic polymers are the most widely employed mucoadhesive vehicles in pharmaceutical formulations. Anionic polymers contain carboxyl and sulphate functional groups that give rise to a net overall negative charge. Examples include poly-acrylic acid (PAA), its weakly cross-linked derivatives and sodium carboxymethylcellulose (NaCMC). Both polymers possess excellent mucoadhesive characteristics because of the formation of hydrogen bonding interactions with mucin from porcine stomach [10].

Due to its good biocompatibility, biodegradability and favorable toxicological properties [11] chitosan is among the most extensively investigated cationic polysaccharides, produced by the deacetylation of chitin [12]. Chitosan binds to mucin via ionic interactions between primary amino functional groups and sialic and sulphonic acid of mucins $[13,14]$. The hydroxyl and amino groups may also interact with mucus via hydrogen bonding.

The major benefit of using chitosan within pharmaceutical applications has been its chemical reactivity and the easiness of addition of various chemical groups, in particular to the C-2 position, allowing the formation of novel polymers with improved functionality. Using such modifications, the properties of chitosan may be tailored to the requirements of specific pharmaceutical-technological challenges [15].

\subsection{Second-Generation Mucoadhesives}

The major disadvantage in using traditional, non-specific mucoadhesive systems is that adhesion may occur at sites different than those intended, i.e. delivery of the drug may occur nonspecifically to any region of the 10-meter long gastrointestinal tract. Unlike first-generation nonspecific polymers, certain second-generation polymers are less susceptible to mucus turnover rates, with a potential to bind directly to mucosal surfaces via the process of "cytoadhesion". Taking into consideration different surface topography of potential target sites, this could be an advantage for the creation of more accurate drug delivery vehicles.

\section{Mucus Structure}

In order to improve bioadhesive properties of drug delivery vehicles it is necessary to reveal structural features of epithelial surfaces for which the drug is targeted to. $\mathrm{Mu}-$ cus is a complex viscous secretion synthesized by specialized goblet cells in the columnar epithelium that lines all of the organs exposed to the external environment. This includes the respiratory tract, the gastrointestinal tract, the reproductive tract, and the oculo-rhino-otolaryngeal tract [16]. Its physiological functions at these locations include maintaining a hydrated layer over the epithelium, shielding the epithelial surfaces against physical and chemical damage, posing a barrier to pathogens and nontoxic substances and acting as a permeable gel layer for the exchange of gases and nutrients with the underlying epithelium $[17,18]$. Mucus is the first barrier with which nutrients and enteric drugs must interact and pass through, in order to be absorbed and to reach the circulatory system and respective target organs. The mucus blanket is highly hydrated (95\% water), with the main component of this extracellular epithelial layer responsible for its viscosity and gel-like properties being the glycoprotein mucin.

Mucins are large ( 0.5 to $20 \mathrm{MDa}$ ) membrane bound and extracellular glycoproteins. They are highly glycolsylated and consist of about $80 \%$ of carbohydrates, primarily N-acetyl-D-galactosamine, N-acetyl-D-glucosamine, fucose, galactose, and sialic acid (N-acetylneuraminic acid), with traces of mannose and sulfate.

The oligosaccharide chains, consisting of 5 - 15 monomers, exhibit moderate branching and are attached to the protein core by O-glycoside bonds via the hydroxyl side chains of serine and threonine and arranged in a "bottle brush" configuration around the protein core. NMR studies of MUC1 secondary structure revealed very little alpha helixes, a small amount of beta sheets and mostly random coil $[19,20]$. Currently, approximately 19 human mucin (designated MUC) genes have been identified, cloned and partially sequenced, and homologs of many of these have been identified in mice and rats [21].

The well-know tendency of other substances to adhere to mucin, known as mucoadhesivity, is not surprising given that this glycoprotein exhibits electrostatic, hydrophobic, and hydrogen bonding interactions [22]. Mucus covers all the organs that are exposed to the external environment and therefore those locations are targets for bioadhesive drug delivery vehicles.

\section{Mucosal Membrane Delivery}

\subsection{Intranasal Delivery}

Intranasal formulation is regarded as a patient-friendly route of drug administration. In terms of pharmacokinetics, the absorption rate is rapid and followed by a faster onset of action compared with oral and intramuscular administration. Due to the high total area which is surrounded by a dense vascular network the nasal mucosa is regarded as an excellent absorptive surface [23]. In addition, the advantage of this route lies in the fact that the hepatic first-pass metabolism is avoided, compared to oral drug administration, in which a clinically significant 
portion of the drug taken is degraded during first-pass metabolism, requiring a higher oral dose for the given effect. Intranasal drugs can be delivered in a variety of formulations that include powders, drops, topical gels, and sprays.

The most commonly employed intranasal pharmaceuticals are solutions of sympathomimetic vasoconstrictors as nasal decongestants. Apart from local effects, the intranasal route of drug administration is often employed for induction of systemic effects [24]. For example, the synthetic hormone desmopressin exerts its action on the kidneys by reducing urine production. It is formulated for intranasal application, frequently prescribed for treatment of Diabetes insipidus.

Another systemic effect achieved by intranasal drug administration is used in the treatment of patients with vitamin B12 deficiency. After normalization with intramuscular vitamin B12 therapy, maintenance of vitamin B12 concentration is achievable with CaloMist (cyanocobalamin) nasal spray that is used on a daily base [25].

\subsection{Gastrointestinal Tract Delivery}

Despite the tremendous advances in drug delivery the oral route still remains the preferred route for the administration of therapeutic agents due to low cost, ease of administration and high levels of patient compliance [26].

However, the delivery of therapeutic agents to, or via the oral cavity is limited by the efficient removal mechanisms that exist in this area. The oral cavity is employed as a site for local and systemic drug delivery [27]. Hepatic first pass metabolism and drug degradation within the gastrointestinal tract are additional obstacles in the oral administration of certain classes of drugs, such as peptides and proteins.

It is generally believed that mucoadhesive drug delivery systems have not reached their full potential within oral drug delivery, since their lack of sufficient adhesion onto the GI tract does not provide prolonged residence time [28]. Targeted drug delivery systems have mainly been focused on mucoadhesive patches and microparticles using first-generation polymers [29]. The problem with mucoadhesive solid formulations, such as tablets, is their poor adherence to mucosal surfaces, combined with their vigorous movement to the GI tract [6]. However, second-generation vehicles have attracted more attention to drug delivery via the GI tract. In an animal model, a thiolated chitosan tablet has been employed for the oral delivery of insulin in rats [30]. In non-diabetic rats a more decreased glucose level was achieved with thiolated chitosan insulin tablets than with unmodified polymer insulin tablets. The explanation for this was that chitosan and the thiol groups showed an inhibitory effect on proteolytic enzymes, together with the penetration enhancing effect of the polymer system, and its improved mucoadhesive potential.

\subsection{Ocular Drug Delivery}

The delivery of aqueous ophthalmic drug solutions shows limitations due to the efficient removal mechanisms that exist within the precorneal area. Ocular drug absorption requires good corneal penetration, along with a prolonged contact time with the corneal tissue [31]. Various approaches have been considered to extend the residence time of topically applied medications in the precorneal region and various formulations such as suspensions, inserts, and aqueous gels have been investigated [32]. It is estimated that almost $95 \%$ of medication delivered by eye drops is lost as the medication mixes with tears and drains into the nasal canal [33]. The first report on the use of soft contact lenses in drug delivery was reported in 1965 for effective treatment of ocular conditions [34]. A drug delivery smart lens has been anticipated to be useful in the control of infection during wound healing following trauma and surgery. Several research groups have been working on strategies for smart lenses that will release medications more evenly over extended periods [35]. One approach in the smart lens technology is based on the suspension of the pharmaceutical in a layer of biodegradable polymer (poly-lactic-co-glycolic acid, PLGA). The relative amount of PLGA to the pharmaceutical will regulate the amount that passes through the lens over time. The more PLGA, the slower the drug is released. In lab tests, these multilayer lenses demonstrated ability to release ciprofloxacin for up to 100 days [36].

\subsection{Buccal Drug Delivery}

The buccal cavity is an attractive target for drug delivery formulations as it is easily accessible. Delivery systems used include mouthwashes, aerosol sprays, chewing gums, bioadhesive tablets, gels and patches [37]. The obstacles associated with drug therapy within the oral cavity are the rapid elimination of drugs due to the flushing effect of saliva, the non-uniform distribution of drug release from a solid or semisolid delivery system within saliva, and the taste and "mouth discomfort" [3841]. In case of side reactions and toxicity buccal drug delivery can be promptly terminated, and therefore this route is considered safe and easy for drug utilization [42]. Due to its unique structural and physiological properties the oral mucosa offers several opportunities for drug delivery. As the mucosa is highly vascularised, any drug diffusing across the oral mucosa membranes has direct access to the systemic circulation and will bypass hepatic circulation. The rate of blood flow through the oral mu- 
cosa is substantial, and is not considered to be the ratelimiting factor in the drug absorption [43]. In addition, saliva a relatively mobile fluid with less mucin and limited enzymatic activity [44], is favorable for protein and peptide delivery.

The first-generation mucoadhesives, such as carboxymethylcellulose [45] have been extensively evaluated mostly for the treatment of periodontal disease [46], and resent research has been mainly focused on the controlled delivery of therapeutic reagents such as peptides, and proteins [47].

\subsection{Vaginal Drug Delivery Systems}

Drug delivery via the vaginal rout is an attractive option due to the avoidance of hepatic first-pass metabolism and a reduction in the incidence of gastrointestinal side effects. The advantages for systemic drug delivery are the large surface area, rich blood supply and high permeability, while low retention time is a disadvantage because of the self-cleansing effect of the vaginal tract [48]. Data from literature demonstrated the superiority of vaginal placement over the oral route regarding minimization of general and gastrointestinal side effects [49]. A very attractive issue concerning the vaginal drug delivery refers to prevention and treatment of sexually transmitted diseases. Some of these approaches will be discussed later.

\section{Lectins as Specific Site Directed Bioadhesives}

Due to the lack of specificity, the first generation of mucoadhesive polymers provides nonspecific binding to mucosal surfaces in the body. Therefore, their employment in the creation of mucoadhesive drug delivery systems for a particular tissue is limited. Creation of polymers and microspheres with attached mucus or cell-specific ligands have increased therapeutic benefits and made site-specific drug delivery possible. In contrast to classical mucoadhesion, which relies on nonspecific interactions of polymer chains and mucins, the lectin interactions with mucins are very specific.

Lectins are a highly heterogeneous group of proteins and glycoproteins of non-immune origin that bind to carbohydrates specifically and noncovalentlly. The term "lectin" derives from the Latin verb legere-to select or choose. Lectins were first discovered in plants; however, their presence was confirmed in most living organisms (bacteria, animals and humans). Lectins are involved in various biological processes: cell-to-cell recognition and communication, particularly in the mammalian immune system (transendothelial migration), adhesion and attack of infectious agents on host cells, clearance of glycoproteins from the blood circulation, etc. [50,51]. The associa- tion constant Ka of lectins with monosaccharides is usu- ally in the range of $10^{3}-10^{4}$ [52]. As carbohydratebinding proteins lectins can increase the adherence of drug delivery vehicles to the mucose surfaces.

The utilization of lectin-mediated drug targeting is based on the fact that most cell surface proteins, and many lipids in the plasma membrane, are glycosylated, and these glycans represent ligands for lectins. Different cell types express various glycan patterns, particularly in pathological states, such as transformed or cancerous cells, where completely different glycans are expressed compared with their healthy counterparts [53]. Therefore, lectins are regarded as potential carrier molecules to target drugs specifically to various cells and tissues. Selection from natural biological material or creation by recombinant DNA technology of a specific lectin, and its coupling to macromolecular drugs or particular drug carriers may lead to efficient cellular uptake and subsequent intracellular routing of such delivery systems. RusselJones and coworkers were able to demonstrate translocation of nanoparticles which had been conjugated to lectins, such as wheat germ agglutinin (WGA), concanavalin A (Con A) and LBT, the binding subunit of heatlabile toxin from E. coli, [54] across the cell layer in an in vitro model of intestinal epithelial cells, whereas A. aurantia lectin, from the edible orange cup mushroom, was able to mediate antigen delivery to M cells [55].

Jain and Jangdey [56] reported on the development of a ConA conjugated gastroretentive multiparticulate delivery system of clarithromycin for the effective treatment of colonization of Helicobacter pylori. Attachment of Con A lectin to ethylcellulose microspheres significantly increased the mucoadhesiveness and also controlled the release of clarithromycin in simulated gastric fluid. Prolonged gastric residence time of over $6 \mathrm{~h}$ was achieved in rabbits for Con A-conjugated microspheres of clarithromycin.

For the patient, the gastrointestinal route is the most convenient and attractive method for systemic delivery of drugs. However it is one of the most challenging routes of administration. The acidity of the gastric juice, as well as the gastric and intestinal enzymes and brush border hydrolases can degrade the drug. Also the viscous mucus blanket overlying the epithelium can limit the absorptive capacity of the cell layer, therefore successful mucosal absorption of drugs requires drug formulations which prolong the residence time at the site of absorption and provide an intimate contact to the absorptive tissue [49]. Among reagents available to direct drugs specifically to the gut epithelium, plant lectins are prime candidates, due to their stability in low $\mathrm{pH}$ conditions and their ability to bind specifically to epithelial cells $[50,57]$. It has been shown that recombinant banana lectin (rBanLec), preserved structural stability and its carbohydrate-bind- 
ing potential under the conditions of simulated gastric fluid and simulated intestinal fluid. In this regard it can be considered as a candidate for the novel bioadhesive lectin-based drug delivery systems to the gastrointestinal tract [58].

A recently published paper on the antiviral activity of BanLec attracted lots of attention even beyond the scientific community, as it has been shown that BanLec has the potential to inhibit genome integration of human immunodeficiency virus (HIV) in the target cell, and hence viral replication [59]. Viruses containing highmannose glycosylated envelopes, such as human immunodeficiency virus type-1 (HIV-1), are potential targets for BanLec binding. The entry of human immunodeficiency virus into cells requires the sequential interactions of the viral exterior envelope glycoprotein, gp120, with the CD4 glycoprotein and a chemokine receptor on the host cell surface. It has been found that BanLec inhibited HIV-1 infection by binding to the glycosylated viral envelope in a way that blocks cellular entry of the virus. The relative anti-HIV activity of BanLec compared favorably to other anti-HIV lectins, such as the snowdrop lectin and griffithisin, and to T-20 and maraviroc, two anti-HIV drugs currently in clinical use. Based on these results, BanLec is a potential component for an anti-viral microbicide that could be used to prevent the sexual transmission of HIV-1. Although concerns have been raised about the potential toxicity of lectins, creation of recombinant therapeutic protein which can be attached to polyethylene glycol (PEG) polymer chains to change bioavailability and reduce toxicity [59].

As ocular drug delivery is limited both by patients' acceptability and by the limited time that the dosage form is retained within the precorneal region, Nicholls et al. [60] tried to identify lectin receptors within the precorneal region as potential targets for a lectin containing ocular dosage form, and thus facilitate prolonged drug delivery. In an ex-vivo experiment conducted on rat corneal and conjunctival intact (unfixed) epithelia, the lectins from Solanum tuberosum (potato) and Helix pomatia (edible snail), with specificity for N-acetyl-D-glucosamine and N-acetyl-D-galactosamine respectively, were the most promising lectins with binding to ocular tissues in terms of a $10 \mathrm{~s}$ contact [60]. Following this, a study which explored the potential of these lectins to cause inflammation and tissue necrosis was performed with New Zealand white rabbits. The authors concluded that Solanum tuberosum and Helix pomatia lectins demonstrated minimal acute irritancy, and would be suitable for formulations and in vivo studies [61].

Beside specific mucoadhesion and cytoadhesion, it has been shown that certain plant lectins are prone to induction of immune response upon oral feeding [62]. In this regard, utilization of novel immunostimulatory molecules having an adjuvant effect to enhance or redirect an immune response against target immunogens has been an important issue in vaccine development. Based on its IgG4-inducing potential, BanLec is regarded as a potentially useful protein carrier for oral antihapten immunization in humans suffering from IgE mediated allergic disorders [63]. Resistance to the proteolytic enzymes of the gastrointestinal tract [64] enables BanLec to pass the mucosal barrier and interact with the immune system, inducing a strong IgG4 immune response.

\section{Conclusions}

In the recent past, new technologies have established DNA arrays that distinguish different cell types through the profiling of gene expression. However, cell surface phenotypes are not only determined by gene expression. Posttranslational modifications by glycosylation and regulation of protein localization are believed to be essential in determining the identity of a particular cell type at a given stage of differentiation. Therefore, it should be possible to distinguish different cell phenotypes through the profiling of cell-surface glycans. In this regard, it seems feasible to design drug delivery vehicles with improved bioadhesive features, and particularly with specific cytoadhesive properties. A very promising concept, which can greatly expand the utility of lectins and afford more accurate and reliable cellular identification and targeting, is the development of novel lectin libraries with diverse specificities [65]. Such an approach offers the possibility of more specific targeting, attachment and controlled release of novel lectin-based vehicles. Despite the promise still lot of investigation has to be conducted in order to produce improved lectin-based therapeutic reagents.

\section{Acknowledgements}

This work is supported by Grant No. 172049, sponsored by the Ministry of Education and Science, Republic of Serbia.

\section{REFERENCES}

[1] A. C. Goddard and D. M. Goddard, "Novel Drug Delivery Systems: Future Directions," Journal of Neuroscience Nursing, Vol. 41, No. 2, 2009, pp. 115-120. doi:10.1097/JNN.0b013e318193458b

[2] A. S. Hoffman, B. D. Ratner, F. J. Schoen and J. E. Lemons, "Biomaterial Science: An Introduction to Materials in Medicine,” 2nd Edition, Elsevier Academic Press, 2004.

[3] T. Nagai and Y. Machida, "Mucosal Adhesive Dosage Forms," Pharmaceutical International, Vol. 6, No. 5, 1985, pp. 196-200. 
[4] K. R. Kamath and K. Park, "Mucosal Adhesive Preparations,” In: J. Swarbrick and J. C. Boylan, Eds., Encyclopedia of Pharmaceutical Technology, Vol. 10, Marcel Dekker, New York, 1994, pp. 133-163.

[5] S. Roy, K. Pal, A. Anis, K. Pramanik and B. Prabhakar, "Polymers in Mucoadhesive Drug Delivery System: A Brief Note,” Designed Monomers and Polymers, Vol. 12, No. 6, 2009, pp. 483-495. doi:10.1163/138577209X12478283327236

[6] G. P. Andrews, T. P. Laverty and D. S. Jones, "Mucoadhesive Polymeric Platforms for Controlled Drug Delivery," European Journal of Pharmaceutics and Biopharmaceutics, Vol. 71, No. 3, 2009, pp. 505-518. doi:10.1016/j.ejpb.2008.09.028

[7] A. Shojaei and X. Li, "Mechanisms of Buccal Mucoadhesion of Novel Copolymers of Acrylic Acid and Polyethylene Glycol Monomethylether Monomethacrylate," Journal of Controlled Release, Vol. 47, No. 2, 1997, pp. 151-161. doi:10.1016/S0168-3659(96)01626-4

[8] K. Park and J. R. Robinson, "Bioadhesive Polymers as Platforms for Oral-Controlled Drug Delivery: Method to Study Bioadhesion," International Journal of Pharmacology, Vol. 19, 1984, pp. 107-127. doi:10.1016/0378-5173(84)90154-6

[9] A. Ludwig, "The Use of Mucoadhesive Polymers in Ocular Drug Delivery,” Advanced Drug Delivery Review, Vol. 57, No. 11, 2005, pp. 1595-1639. doi:10.1016/j.addr.2005.07.005

[10] N. Fefelova, Z. Nurkeeva, G. Mun and V. Khutoryanskiy, "Mucoadhesive Interactions of Amphiphilic Cationic Copolymers Based on [2-(Methacryloyloxy)ethyl]trimethylammonium Chloride,” International Journal of Pharmacology, Vol. 339, 2007, pp. 25-32.

[11] A. Portero, D. Teijeiro-Osorio, M. Alonso and C. Remuñán-López, "Development of Chitosan Sponges for Buccal Administration of Insulin," Carbohydrate Polymers, Vol. 68, No. 4, 2007, pp. 617-625. doi:10.1016/j.carbpol.2006.07.028

[12] P. He, S. Davis and L. Illum, "In Vitro Evaluation of the Mucoadhesive Properties of Chitosan Microspheres," International Journal of Pharmacology, Vol. 166, No. 1, 1998, pp. 75-88. doi:10.1016/S0378-5173(98)00027-1

[13] S. Rossi, F. Ferrari, M. Bonferoni and C. Caramella, "Characterization of Chitosan Hydrochloride-Mucin Interaction by Means of Viscosimetric and Turbidimetric Measurements," European Journal of Pharmaceutical Science, Vol. 10, No. 4, 2000, pp. 251-257. doi:10.1016/S0928-0987(00)00065-8

[14] A. Bernkop-Schnürch, "Mucoadhesive Systems in Oral Drug Delivery,” Drug Discovery Today: Technology, Vol. 2, No. 1, 2005, pp. 83-87. doi:10.1016/j.ddtec.2005.05.001

[15] A. Bernkop-Schnurch, "Chitosan and Its Derivatives: Potential Excipients for Peroral Peptide Delivery Systems," International Journal of Pharmaceutics, Vol. 194, No. 1, 2000, pp. 1-13. doi:10.1016/S0378-5173(99)00365-8
[16] R. Bansil and B. S. Bradley, "Mucin Structure, Aggregation, Physiological Functions and Biomedical Applications," Current Opinion in Colloid \& Interface Science, Vol. 11, No. 2-3, 2006, pp. 164-170. doi:10.1016/j.cocis.2005.11.001

[17] A. Allen, "Structure and Function of Gastrointestinal Mucus,” In: L. Johnson, Ed., Physiology of the Gastroenterology Tract, Raven Press, New York, 1981, pp. 617639.

[18] M. Neutra and J. Forstner, "Gastrointestinal Mucus: Synthesis, Secretion, and Function,” In: L. Johnson, Ed. Physiology of the Gastrointestinal Tract, Raven Press, New York, Chapter 34, 1987.

[19] J. Fontenot, N. Tjandra, D. Bu, C. Ho, R. Montelaro and O. Finn, "Biophysical Characterization of One-, Two-, and Three-Tandem Repeats of Human Mucin (Muc-1) Protein Core," Cancer Research, Vol. 53, 1993, pp. 8694.

[20] L. Otvos and M. Cudic, "Conformation of Glycopeptides,” Mini Review in Medicinal Chemistry, Vol. 3, No. 7, 2003, pp. 703-711. doi:10.2174/1389557033487809

[21] J. Perez-Vilar and R. Hill, "The Structure and Assembly of Secreted Mucins," Journal of Biological Chemistry, Vol. 274, No. 45, 1999, pp. 31751-31754. doi:10.1074/jbc. 274.45.31751

[22] S. Harding, S. Davis, M. Deacon and I. Fiebrig, "Biopolymer Mucoadhesives,” Biopolymer Mucoadhesives, Vol. 16, 1999, pp. 41-86.

[23] J. M. Gu, J. R. Robinson and S. H. Leung, "Binding of Acrylic Polymers to Mucin/Epithelial Surfaces: Structure-Property Relationships," Critical Reviews in Therapeutic Drug Carrier Systems, Vol. 5, No. 1, 1988, pp. 2167.

[24] H. Costantino, L. Illum, G. Brandt and P. S. Johnson, "Quay, Intranasal Delivery: Physicochemical and Therapeutic Aspects,” International Journal of Pharmacology, Vol. 337, 2007, pp. 1-24. doi:10.1016/j.ijpharm.2007.03.025

[25] D. M. Suzuki, K. Alaqiakrishnan, K. H. Masaki, A. Okada and M. Carethers, "Patient Acceptance of Intranasal Cobalamin Gel for Vitamin B12 Replacement Therapy,” Hawaii Medical Journal, Vol. 65, No. 11, 2006, pp. 311-314.

[26] V. F. Patel, F. Liu and M. B. Brown, “Advances in Oral Transmucosal Drug Delivery,” Journal of Controlled Release, Vol. 153, No. 2, 2011, pp. 106-116. doi:10.1016/j.jconrel.2011.01.027

[27] J. D. Smart, "Lectin-Mediated Drug Delivery in the Oral Cavity,” Advanced Drug Delivery Reviews, Vol. 56, No. 4, 2004, pp. 481-489. doi:10.1016/j.addr.2003.10.016

[28] M. Chun, H. Sah and H. Choi, "Preparation of Mucoadhesive Microspheres Containing Antimicrobial Agents for Eradication of H. Pylori,” International Journal of Pharmacology, Vol. 297, No. 1-2, 2005, pp. 172-179.

[29] M. Säkkinen, J. Marvola, H. Kanerva, K. Lindevall, A. Ahonen and M. Marvola, "Are Chitosan Formulations 
Mucoadhesive in the Human Small Intestine? An Evaluation Based on Gamma Scintigraphy," International Journal of Pharmacology, Vol. 307, No. 2, 2006, pp. 285-291.

[30] A. Krauland, D. Guggi and A. Bernkop-Schnürch, “Oral Insulin Delivery: The Potential of Thiolated ChitosanInsulin Tablets on Non-Diabetic Rats," Journal of Controlled Release, Vol. 95, No. 3, 2004, pp. 547-555. doi:10.1016/j.jconrel.2003.12.017

[31] D. L. Middleton, S. H. S. Leung and J. R. Robinson, "Ocular Bioadhesive Delivery Systems,” In: V. Lenaerts and R. Gurny, Eds., Bioadhesive Drug Delivery Systems, CRC Press, Florida, 1990, pp. 180-201.

[32] J. L. Greaves, “Ocular Drug Delivery,” In: C. G. Wilson and N. Washington, Eds., Physiological Pharmaceuticals, Ellis Horwood, Chichester, 1989, pp. 121-138.

[33] in-Pharma Technologist.com, "Contact Lenses Deliver Drugs to Eye,” 10 January 2005, Retrieved on 30 May 2008.

http://www.in-pharmatechnologist.com/news/ng.asp?id=5 7187-contact-lenses-deliver

[34] J. Sedlavek, "Possibilities of Application of Ophthalmic Drugs with the Aid of Gel Contact Lenses," Cesk Oftalmology, Vol. 21, 1965, pp. 509-514.

[35] J. A. Legerton, “The future Is Now: Unveiling Smart Lenses," http://www.reviewofcontactlenses.com/content/c/28320/

[36] J. B. Ciolino, T. R. Hoare, N. G. Iwata, I. Behlau, C. H. Dohlman, R. Langer and D. S. Kohane, “A Drug-Eluting Contact Lens,” Investigative Ophthlamology \& Visual Science, Vol. 50, No. 7, 2009, pp.3346-52. doi:10.1167/iovs.08-2826

[37] D. Steinbers and M. Friedman, "Dental Drug-Delivery Devices: Local and Sustained-Release Applications," Critical Review in Therapeutic Drug Carrier Systems, Vol. 16, No. 5, 1999, pp. 425-459.

[38] D. Harris and J. R. Robinson, "Drug Delivery via the Mucous Membranes of the Oral Cavity," Journal of Pharmaceutical Sciences, Vol. 81, No. 8, 1992, pp. 1-10. doi:10.1002/jps.2600810102

[39] M. J. Rathbone and I. G. Drummond, "The Oral Cavity as a Site for Systemic Drug Delivery,” Advances Drug Delivery Review, Vol. 13, No. 1-2, 1994, pp. 1-22. doi:10.1016/0169-409X(94)90024-8

[40] J. D. Smart, "Drug Delivery Using Buccal Adhesive Systems,” Advances Drug Delivery Review, Vol. 11, No. 3, 1993, pp. 253-270.

doi:10.1016/0169-409X(93)90012-S

[41] J. A. Wheatherell, C. Robinson and M. J. Rathbone, "Side Specific Differences in the Salivary Concentrations of Substances in the Oral Cavity-Implications for the Aetiology of Oral Disease and Local Drug Delivery,” Advances Drug Delivery Review, Vol. 13, No. 1-2, 1994, pp. 23-42. doi:10.1016/0169-409X(94)90025-6

[42] V. M. Patel, B. G. Prajapati and M. M. Patel, "Formulation, Evaluation, and Comparison of Bilayered and Multilayered Mucoadhesive Buccal Devices of Propranolol Hydrochloride,” AAPS Pharmscitech, Vol. 8, No. 1, 2007,

\section{pp. E147-E154. doi:10.1208/pt0801022}

[43] V. F. Patel, F. Liu and M. C. Brown, "Advances in Oral Transmucosal Drug Delivery,” Journal of Controlled Release, Vol. 153, No. 2, 2011, pp. 106-116. doi:10.1016/j.jconrel.2011.01.027

[44] Y. Huang, W. Leobandung, A. Foss and N. A. Peppas, "Molecular Aspects of Muco- and Bioadhesion: Tethered Structures and Site-Specific Surfaces," Journal of Controlled Release, Vol. 65, No. 1-2, 2000, pp. 63-71. doi:10.1016/S0168-3659(99)00233-3

[45] N. Fefelova, Z. Nurkeeva, G. Mun and V. Khutoryanski, "Mucoadhesive Interactions of Amphiphilic Cationic Copolymers Based on [2-(Methacryloyloxy) ethyl trimethyl] Ammonium Chloride," International Journal of Pharmaceutics, Vol. 339, No. 1-2, 2007, pp. 25-32. doi:10.1016/j.ijpharm.2007.02.019

[46] D. Jones, A. D. Woolfson, A. Brown, W. Coulter, C. McClelland and C. Irwin, "Design, Characterization and Preliminary Clinical Evaluation of a Novel Mucoadhesive Topical Formulation Containing Tetracycline for the Treatment of Periodontal Disease," Journal of Controlled Release, Vol. 67, No. 2-3, 2000, pp. 357-368. doi:10.1016/S0168-3659(00)00231-5

[47] H. Junginger, J. Hoogstraate and J. C. Verhoef, "Recent Advances in Buccal Drug Delivery and Absorption-In Vitro and in Vivo Studies," Journal of Controlled Release, Vol. 62, No. 1, 1999, pp. 149-159. doi:10.1016/S0168-3659(99)00032-2

[48] Z. Pavelic, N. Skalko-Basnet and R. Schubert, "Liposomal Gels for Vaginal Drug Delivery,” International Journal of Pharmacology, Vol. 219, No. 1-2, 2001, pp. 139-149. doi:10.1016/S0378-5173(01)00637-8

[49] A. Darwish, E. Hafez, I. El-Gebali and S. Hassan, "Evaluation of a Novel Vaginal Bromocriptine Mesylate Formulation: A Pilot Study,” Fertility and Sterility, Vol. 83, No. 4, 2005, pp. 1053-1055. doi:10.1016/j.fertnstert.2004.09.024

[50] F. Gabor, E. Bogner, A. Weissenboeck and M. Wirth, "The Lectin-Cell Interaction and Its Implications to Intestinal Lectin Mediated Drug Delivery,” Advanced Drug Delivery Reviews, Vol. 56, No. 4, 2004, pp. 459-480. doi:10.1016/j.addr.2003.10.015

[51] M. Gavrovic-Jankulovic and M. Groydanovic, “Application of Plant-Derived Food Lectins in Proteoglycomics and Immunomodulation," In: S. Haugen and S. Meijer, Eds., Handbook of Nutritional Biochemistry: Genomics, Metabolomics, and Food Supply, Nova Science Publishers, Inc, New York, 2010, pp. 405-423.

[52] H. Lis, D. Belenky, A. Rabinkov and N. Sharon, "Purification of Lectins and Determination of Their Carbohydrate Specificity,” In: J. E. Celis, Ed., Cell Biology-A Laboratory Handbook, Academic Press, San Diego, 1994, pp. 332-338.

[53] C. Bies, C. M. Lehr and J. F. Woodley "Lectin-Mediated Drug Targeting: History and Applications,” Advanced Drug Delivery Reviews, Vol. 56, 2004, pp. 425-435. doi:10.1016/j.addr.2003.10.030 
[54] G. J. Russel-Jones, H. Veitch and L. Arthur, "LectinMediated Transport of Nanoparticles across Caco-2 and OK Cells,” International Journal of Pharmaceutics, Vol. 190, No. 2, 1999, pp. 165-174. doi:10.1016/S0378-5173(99)00254-9

[55] F. Roth-Walter, I. Schöll, E. Untersmayr, R. Fuchs, G. Boltz-Nitulescu, A. Weissenbock, O. Scheiner, F. Gabor and E. Jensen-Jarolim, "M Cell Targeting with Aleuria Aurantia Lectin as a Novel Approach for Oral Allergen Immunotherapy," Journal of Allergy and Clinical Immunology, Vol. 114, No. 6, 2004, pp. 1362-1368. doi:10.1016/j.jaci.2004.08.010

[56] S. K. Jain and M. S. Jangdey, "Lectin Conjugated Gastroretentive Multiparticulate Delivery System of Clarithromycin for the Effective Treatment of Helicobacter Pylori,” Molecular Pharmaceutics, Vol. 6, 2008, pp. 295304. doi:10.1021/mp800193n

[57] E. C. Lavelle, "Targeted Delivery of Drugs to the Gastrointestinal Tract," Critical Reviews in Therapeutic Drug Carrier Systems, Vol. 18, No. 4, 2001, pp. 341-386.

[58] R. Dimitrijevic, M. Jadranin, L. Burazer, S. Ostojic and M. Gavrovic-Jankulovic, "Evaluation of the Thermal Stability and Digestibility of Heterologously Produced Banana Lectin,” Food Chemistry, Vol. 120, 2010, pp. 11131118. doi:10.1016/j.foodchem.2009.11.062

[59] M. Swanson, H. Winter, I. Goldstein and D. Markovitz, "A Lectin Isolated from Bananas Is a Potent Inhibitor of HIV Replication,” Journal of Biological Chemistry, Vol. 285, No. 12, 2010, pp. 8646-8655. doi:10.1074/jbc.M109.034926

[60] J. N. Tanya, K. L. Green, D. J. Rogers, J. D. Cook, S.
Wolowacz and J. D. Smart, "Lectins in Ocular Drug Delivery: An Investigation of Lectin Binding Sites on the Corneal and Conjunctival Surfaces,” International Journal of Pharmaceutics, Vol. 138, No. 2, 1996, pp. 175-183. doi:10.1016/0378-5173(96)04540-1

[61] J. D. Smart, T. J. Nicholls, K. L. Green, D. J. Rogers and J. D. Cook, "Lectins in Drug Delivery: A Study of the Acute Local Irritancy of the Lectins from Solanum Tuberosum and Helix Pomatia," European Journal of Pharmaceutical Sciences, Vol. 9, No. 1, 1999, pp. 93-98. doi:10.1016/S0928-0987(99)00050-0

[62] H. J. de Aizpurua and G. J. R. Jones, “Oral Vaccination: Identification of Classes of Proteins That Provoke an Immune Response upon Oral Feeding," The Journal of Experimental Medicine, Vol. 167, 1988, pp. 440-451. doi:10.1084/jem.167.2.440

[63] M. D. Chapman, A. M Smith, L. D Vailes, L. K Arruda, V. Dhanaraj and A. Pomes, "Recombinant Allergens for Diagnosis and Therapy of Allergic Diseases," Journal of Allergy and Clinical Immunology, Vol. 106, No. 3, 2000, pp. 409-418. doi:10.1067/mai.2000.109832

[64] W. J. Peumans, W. Zhang, A. Barre, C. H. Astoul, P. J. Balint-Kurti, P. Rovira, G. D. May, F. Van Leuven, P. Truffa-Bachi and E. J. Van Damme, "Fruit-Specific Lectins from Banana and Plantain,” Planta, Vol. 211, No. 4, 2000, pp. 546-554. doi:10.1007/s004250000307

[65] M. Yim, T. Ono and T. Irimura, "Mutated Plant Lectin Library Useful to Identify Different Cells,” Proceedings of National Academy of Sciences, Vol. 98, No. 5, 2001, pp. 2222-2225. doi:10.1073/pnas.041621998 